\title{
Parteimitgliedschaften im Jahre 2011
}

\section{Oskar Niedermayer}

Mit der folgenden Dokumentation wird der seit 2001 jährlich erscheinende systematische Überblick über die Entwicklung, regionale Verteilung und sozialstrukturelle Zusammensetzung der Parteimitgliedschaften fortgeschrieben. Zusätzliche Schaubilder sind über das Internet abrufbar (http://www.polsoz.fu-berlin.de/polwiss/forschung/systeme/empsoz/schriften/Arbeitshefte/ahosz19.pdf). ${ }^{1}$ Es werden nur Daten aus der Mitgliederverwaltung der Parteien verwendet. ${ }^{2}$ Auf Angaben über die berufliche Stellung wird verzichtet, weil die hierzu vorhandenen Daten den Beruf zum Zeitpunkt des Parteieintritts ohne spätere Aktualisierung wiedergeben und somit die tatsächliche Berufsstruktur der Parteimitgliedschaften nicht korrekt widerspiegeln.

Im Herbst 2008 wurde auf Anregung der Bundesgeschäftsführer vom Verfasser ein Workshop mit allen für die Mitgliederstatistik verantwortlichen Mitarbeitern der Parteien durchgeführt, auf dem eine Reihe von Verbesserungen der Mitgliederstatistik und ihrer Publikation beschlossen wurde. Daher gibt es seit Ende 2008 eine Altersstatistik der Mitglieder mit einer identischen, sehr feinen Altersgruppeneinteilung sowie eine Statistik der Eintritte, Austritte und Todesfälle und der Eintritte nach Geschlecht und Alter. Zusätzlich werden Daten zur Rekrutierungsfähigkeit der Parteien und zur Über- beziehungsweise Unterrepräsentation bestimmter Bevölkerungsgruppen in den Parteimitgliedschaften publiziert.

Trotz des Bemühens aller Parteien, ihre Mitgliedschaft wenigstens zu stabilisieren, mussten alle bis auf die Grünen im Jahre 2011 mehr oder minder große Verluste hinnehmen. Am wenigsten verlor die CSU. Im Vergleich der beiden Großparteien waren die CDU-Verluste das zweite Jahr in Folge größer als die Verluste der SPD. Die Linkspartei, die nach ihrer Gründung 2007 (Zusammenschluss aus PDS und WASG) zunächst Mitgliedergewinne verzeichnen konnte, verlor in den letzten beiden Jahren jeweils zwischen fünf und sechs Prozent ihrer Mitgliedschaft. Am schlimmsten traf es die FDP, deren Absturz in der Wählergunst in den letzten beiden Jahren sich auch in einem deutlichen Mitgliederrückgang niederschlug (2011 fast acht Prozent). Für die Grünen hingegen brachte der demoskopische Höhenflug 2010/2011 mit zehn Prozent beziehungsweise 11,5 Prozent die stärksten Mitgliederzuwächse seit Mitte der Achtzigerjahre. Über den gesamten Zeitraum seit 1990 haben alle Parteien außer den Grünen Mitglieder verloren, wenn auch in sehr unterschiedlichem Maße. Am stärksten hat es die Linke getroffen, die - trotz des Zuwachses durch die Vereinigung von PDS und WASG - Ende 2011 mehr als drei Viertel weniger Mitglieder zählt als die PDS Ende 1990. Die FDP hat seit 1990 mehr als drei Fünftel ihrer Mitglieder verloren, die SPD fast die Hälfte, die CDU fast zwei Fünftel und die CSU fast ein Fünftel. Die Grünen hingegen konnten ihre Mitgliedschaft seit 1990 um 43 Prozent steigern (vgl. Tabelle 1).

1 Für eine Analyse der Daten seit 1946 vgl. Oskar Niedermayer, Der Wandel des parteipolitischen Engagements der Bürger, in: Steffen Kühnel / Oskar Niedermayer / Bettina Westle (Hrsg.), Wähler in Deutschland. Sozialer und politischer Wandel, Gender und Wahlverhalten, Wiesbaden 2009, S. $82-134$.

2 Den Geschäftsstellen der Parteien, die dem Verfasser die Daten freundlicherweise zur Verfügung gestellt haben, sei an dieser Stelle ausdrücklich gedankt. 
Betrachtet man statt der absoluten Mitgliederzahlen die Rekrutierungsfähigkeit - also den Anteil der Parteimitglieder an den Beitrittsberechtigten - dann verändern sich die Größenordnungen zwischen den Parteien, da sie unterschiedliche Mindestalterbestimmungen für den Parteibeitritt haben (ab 14 beziehungsweise 16 Jahren) und die CDU ihre Mitglieder nur außerhalb Bayerns rekrutieren kann, während die CSU auf Bayern beschränkt ist. Die SPD war bis zum Frühjahr 2008 die mitgliederstärkste Partei. Hinsichtlich der Fähigkeit zur Mitgliederrekrutierung wurde sie allerdings schon 1999 von der CDU überholt, wobei beide Parteien wiederum weit hinter der CSU zurückliegen, deren Mitgliedschaft Ende 2010 mehr als 1,4 Prozent der bayerischen Bevölkerung ab 16 Jahren umfasste, während die SPD nur 0,7 Prozent der gesamtdeutschen Bevölkerung ab 14 Jahren für eine SPD-Mitgliedschaft gewinnen konnte. Insgesamt waren 1980, nach dem Hinzukommen der Grünen, in der alten Bundesrepublik fast 4 Prozent der beitrittsberechtigten Bevölkerung (knapp zwei Millionen Bürger) in einer der fünf Parteien organisiert. Ende 1989 waren es noch 3,6 Prozent, nach der Vereinigung und dem Hinzukommen der damaligen PDS 3,7 Prozent. Ende 2010 gehörten nur noch 1,9 Prozent der beitrittsberechtigten Bevölkerung einer der sechs wichtigen deutschen Parteien an (für Ende 2011 lagen bei Redaktionsschluss noch keine Bevölkerungsdaten vor). Es ist somit eine kontinuierlich abnehmende gesellschaftliche Verankerung des Parteiensystems zu beobachten (vgl. Tabelle 2).

Die CDU musste im Jahre 2011 Mitgliederverluste in allen Bundesländern hinnehmen, die SPD konnte in den drei Stadtstaaten und Mecklenburg-Vorpommern leichte Zugewinne verbuchen. Bei der FDP schlug sich der Absturz in der Wählergunst auch in den Mitgliederzahlen nieder: Sie verlor in allen Bundesländern Mitglieder, in Bremen, dem Saarland und Sachsen-Anhalt sogar im zweistelligen Prozentbereich. Die Grünen hingegen erzielten überall Zuwächse, die sich in zwei Dritteln der Länder im zweistelligen Prozentbereich bewegten. Bei der Linkspartei setzte sich der flächendeckende Mitgliederschwund der letzten Jahre in Ostdeutschland auch 2011 fort; in den meisten westdeutschen Ländern mussten ebenfalls Verluste hingenommen werden (vgl. Tabelle 3).

Bei der Rekrutierungsfähigkeit der Parteien nach Bundesländern zeigte sich auch 2010 das schon seit längerer Zeit bestehende Muster: Bei der SPD und den Grünen bilden die fünf ostdeutschen Bundesländer mit deutlichem Abstand zu allen westdeutschen Ländern das Schlusslicht, und bei der CDU weisen nur die Stadtstaaten Berlin, Bremen und Hamburg ähnlich niedrige Rekrutierungsgrade auf wie die ostdeutschen Länder. Bei der FDP bestehen hingegen keine systematischen Ost-West-Unterschiede. Die Linke blieb auch 2010 von ihrer Mitgliederverankerung her noch eine ostdeutsche Regionalpartei, die im Westen - mit Ausnahme des Saarlands, der Heimat von Oskar Lafontaine - eine geringe Organisationsbasis aufwies; sie erreichte aber auch im Osten nirgendwo auch nur annähernd die Rekrutierungsfähigkeit der CDU, SPD und CSU in ihren westdeutschen Hochburgen (vgl. Tabelle 4).

Beim Anteil der Frauen an den Parteimitgliedschaften ist über einen längeren Zeitraum kein parteiübergreifender Trend festzustellen. Bei CDU, SPD und der Linkspartei blieb er 2011 gegenüber 2010 konstant, bei den anderen Parteien erhöhte er sich leicht (vgl. Tabelle 5).

Der Anteil einer bestimmten Bevölkerungsgruppe unter den Parteimitgliedern sagt noch nichts darüber aus, ob diese Gruppe in den Parteimitgliedschaften gegenüber der Bevölkerung unter- oder überrepräsentiert ist. Um die Art der Repräsentation sozialer Gruppen in den einzelnen Parteimitgliedschaften feststellen und zwischen den Parteien exakt vergleichen 
zu können, wurden daher so genannte Proportionalitätsquotienten (PQ) gebildet, indem der Anteil einer Gruppe an den Parteimitgliedern durch den Anteil dieser Gruppe an der jeweiligen beitrittsberechtigten Bevölkerung dividiert wurde. PQ-Werte über 1 bedeuten daher eine Überrepräsentation der Gruppe bei den Parteimitgliedern und PQ-Werte unter 1 eine Unterrepräsentation, die umso stärker ist, je kleiner der Wert ist. Die PQ-Werte für den Frauenanteil zeigen, dass die Frauen in den Mitgliedschaften aller Parteien unterrepräsentiert sind, allerdings in deutlich unterschiedlichem Maße: Ende 2010 war zum Beispiel der Anteil der Frauen an der CDU-Mitgliedschaft (25,6 Prozent) halb so groß wie in der Bevölkerung ab 16 Jahren außerhalb Bayerns (51,3 Prozent), so dass eine Unterrepräsentation von $\mathrm{PQ}=0,5$ vorlag. Am stärksten ist die Unterrepräsentation von Frauen in der CSU; in der PDS war sie bis 2006 am geringsten, die Vereinigung mit der WASG hat sie jedoch verstärkt, und seit 2008 ist die Linke mit den Grünen auf gleichem Niveau (vgl. Tabelle 6).

Betrachtet man die Entwicklung der Altersstruktur der Parteimitgliedschaften in der groben Unterteilung in Jüngere (bis 29/30 Jahre), eine mittlere Kategorie (29/30 bis 59/60 Jahre) und Ältere (ab 60/61 Jahre) bis 2007, so ist wohl der bis etwa zur Jahrhundertwende zu beobachtende Rückgang des Anteils der jüngeren Parteimitglieder vor allem für die SPD endgültig gestoppt, denn hier zeigte sich über mehrere Jahre hinweg eine Verbesserung, während die Werte für die CDU und vor allem auch für die FDP in den letzten Jahren vor 2007 wieder abwärts gingen. Den höchsten Anteil an jüngeren Parteimitgliedern wiesen 2007 die Grünen auf, die nach einer Auswertung ihrer Mitgliederdatei erstmals über Daten zur Altersstruktur verfügten. Der geringste Anteil an Jüngeren und der mit Abstand höchste Anteil an Älteren fand sich bis 2006 in der PDS. Durch den Zusammenschluss mit der WASG hat sich die Partei allerdings deutlich „verjüngt“ (vgl. Tabelle 7).

In allen Parteien waren die Jüngeren bis 2007 deutlich unterrepräsentiert und die Älteren überrepräsentiert, wobei sich letzteres über die Jahre deutlich verstärkt hat. Die Unterrepräsentation der Jüngeren hat hingegen nicht in der Weise zugenommen, wie es die Entwicklung der Anteile der Jüngeren an den Mitgliedschaften vermuten lässt, da der Anteil der Jüngeren an der beitrittsberechtigten Bevölkerung im Zeitablauf auch abgenommen hat. So waren zum Beispiel 1990 sieben Prozent der CSU-Mitgliedschaft 16 bis 30 Jahre und 2007 nur noch 5,5 Prozent. Da jedoch der Anteil der 16- bis 30-Jährigen an der bayerischen Bevölkerung ab 16 Jahren 1990 28,3 Prozent und 2007 nur noch 21,1 Prozent betrug, war die Unterrepräsentation der Jüngeren in der CSU 1990 genauso groß wie 2007: PQ 1990 $=0,25,2007=0,26(\mathrm{vgl}$. Tabelle 8).

Die neue Alterstabelle ab 2008 mit vollständiger Vergleichbarkeit und feinerer Gliederung der Alterskategorien zeigt, dass eine Herabsetzung des Beitrittsalters unter 16 Jahre nur sehr wenige Mitglieder bringt. Auch der Anteil der Mitglieder bis 20 Jahre ist bei CDU, SPD, CSU und der Linkspartei noch sehr gering und liegt deutlich unter dem der über 85-Jährigen. Der Anteil der jüngeren Mitglieder (bis 30 Jahre) stagnierte 2008 bis 2011 bei der CDU bei etwa sechs Prozent, bei der CSU ist nach einem zwischenzeitlichen Rückgang wieder eine Steigerung auf über fünf Prozent festzustellen, bei der SPD ist er von 6,8 auf 7,5 Prozent gestiegen. Bei der FDP stellten die Jüngeren 2008 bis 2010 über 12 Prozent der Mitgliedschaft, 2011 ist der Wert auf 11,4 Prozent gefallen. Bei der Linken stieg der Anteil in diesem Zeitraum von 6,4 auf 9,6 Prozent und bei den Grünen von 13,7 auf 16,4 Prozent. Die Linke weist 2011 mit 60 Jahren das höchste Durchschnittsalter der Parteimitglieder auf, gefolgt von CDU und SPD mit 59 Jahren. Die Grünen sind mit einem Durchschnittsalter von 47 Jahren die ,jüngste“ Partei (vgl. Tabelle 9). 
Auch bei den Grünen sind jedoch die 14- bis 20-Jährigen gegenüber der Bevölkerung sehr stark unterrepräsentiert und selbst der Anteil der 21- bis 25-Jährigen an den Parteimitgliedern ist noch kleiner als in der Bevölkerung $(\mathrm{PQ}<1)$. Allerdings setzt sich die Unterrepräsentation bei CDU, SPD und der Linkspartei noch bis einschließlich der Altersgruppe der 46- bis 50-Jährigen fort. In der Mitgliedschaft von CDU, SPD, CSU und der Linkspartei sind die Älteren (ab 61 Jahre) deutlich überrepräsentiert, in der FDP-Mitgliedschaft entsprechen sie in etwa dem Anteil unter den Beitrittsberechtigten, während sie in der GrünenMitgliedschaft deutlich unterrepräsentiert sind (vgl. Tabelle 10).

Für die CDU ist seit Mitte der Neunzigerjahre ein kontinuierlicher Rückgang des Anteils konfessionsgebundener Mitglieder festzustellen, der sich auch 2011 fortgesetzt hat. Dabei hat sich der Protestantenanteil deutlicher verringert als jener der Katholiken. Bei der CSU schwanken die Werte etwas stärker, auch hier geht jedoch der Anteil an Katholiken und Protestanten zurück, wenn auch deutlich langsamer als bei der CDU. Allerdings waren die Katholiken von Anfang an in der Mitgliedschaft beider Parteien überrepräsentiert, und da ihr Anteil in der Bevölkerung im Zeitverlauf noch etwas stärker abgenommen hat als in den Parteimitgliedschaften, hat sich die Überrepräsentation der Katholiken in der CDU und CSU in den letzten zwei Jahrzehnten nicht verringert, sondern sogar noch etwas erhöht. Der Katholikenanteil der CDU in Ostdeutschland ist zwar deutlich geringer als im Westen; setzt man ihn jedoch in Relation zum Katholikenanteil in der Bevölkerung, so zeigt sich, dass die Katholiken in der ostdeutschen CDU-Mitgliedschaft noch weit stärker überrepräsentiert sind als im Westen (vgl. Tabelle 11).

Die ab 2008 zur Verfügung stehenden Daten über Eintritte, Austritte und Todesfälle im Laufe des Jahres zeigen, dass bei CDU und SPD in diesen Jahren jeweils weniger als vier Prozent und bei der CSU weniger als drei Prozent neue Mitglieder hinzukamen. Deutlich größere Neumitgliederquoten hatten FDP, Grüne und die Linkspartei. Die FDP erreichte im Bundestagswahljahr 2009 mit fast 16 Prozent einen sehr hohen Wert, der in den nächsten beiden Jahren jedoch dramatisch einbrach. Die Grünen verzeichneten schon 2010 mit fast 15 Prozent einen sehr hohen Zuwachs und konnten ihn 2011 noch auf über 16 Prozent steigern. Die Eintrittsquoten der Linkspartei verringerten sich seit 2008 mit fast 14 Prozent Neumitgliedern kontinuierlich. Die Parteiaustritte bewegten sich in der Regel in der Größenordnung zwischen 2,5 und fünf Prozent; die FDP musste allerdings 2010 mit 7,7 und 2011 mit 10,5 Prozent deutlich höhere Austrittsquoten der Mitgliedschaft verkraften. Zudem verloren alle Parteien - je nach Grad der Überalterung ihrer Mitgliedschaft - zwischen 0,2 und 2,3 Prozent ihrer Mitglieder pro Jahr durch Todesfälle (vgl. Tabelle 12).

Der Frauenanteil bei den Eintritten schwankt und liegt 2011 bei CDU, FDP und vor allem bei der CSU über dem Frauenanteil der Gesamtmitgliedschaft (vgl. Tabelle 13). Die Altersstruktur der Eintritte ist gegenüber der Gesamtmitgliedschaft wesentlich verjüngt (vgl. die Tabellen 14 und 15). Besonders deutlich zeigt sich dies bei der SPD: Während zum Beispiel Ende 2011 weniger als vier Prozent der Gesamtmitgliedschaft bis 25 Jahre alt waren, betrug der Anteil dieser Altersgruppe unter den Neumitgliedern des Jahres $2011 \mathrm{mehr}$ als 30 Prozent, bei den anderen Parteien nur 15 bis 23 Prozent. Wenn sich dieser Trend fortsetzt, kann die SPD auf eine allmähliche leichte „Verjüngung“ hoffen. 


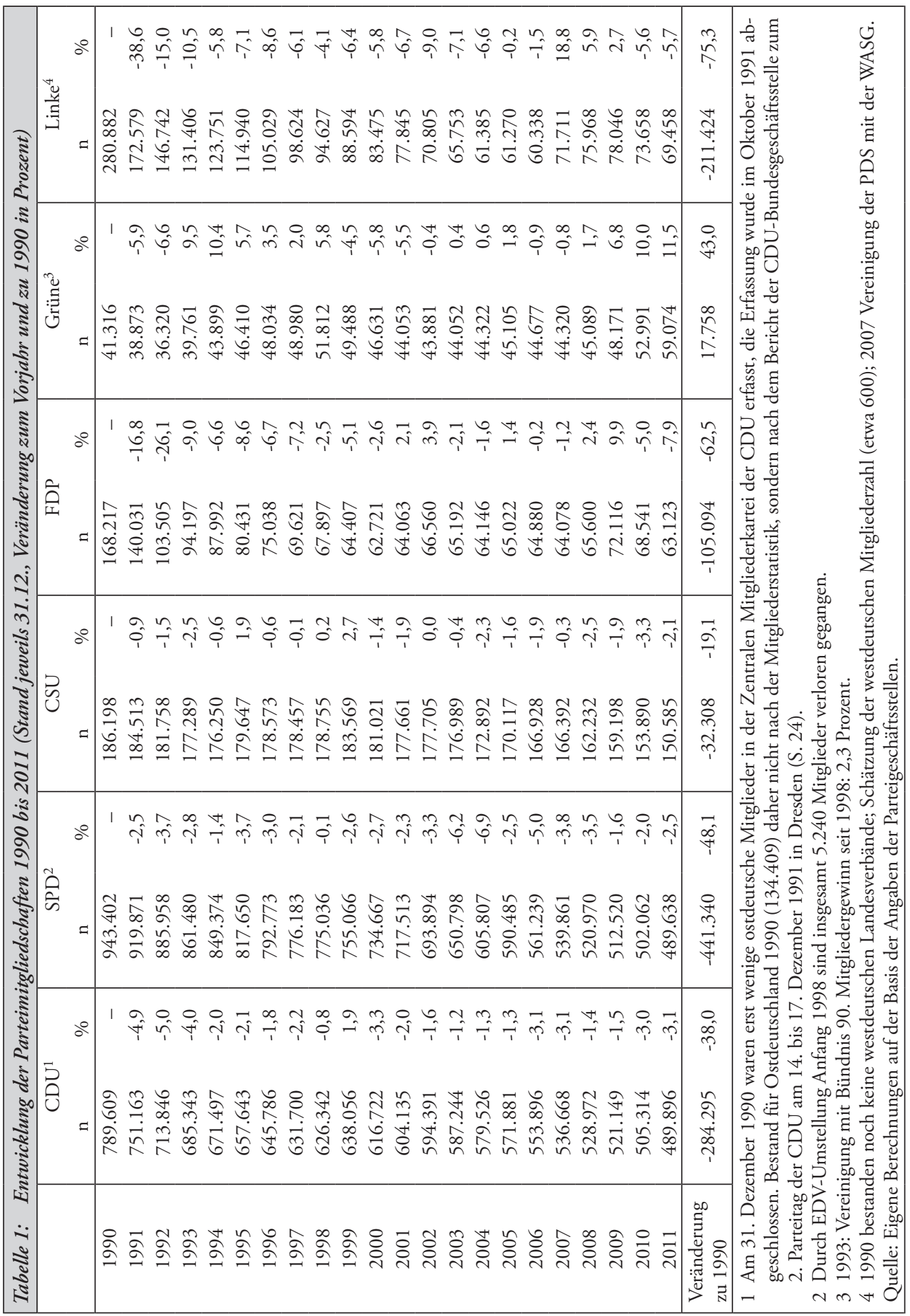




\begin{tabular}{|c|c|c|c|}
\hline & 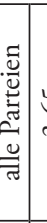 & 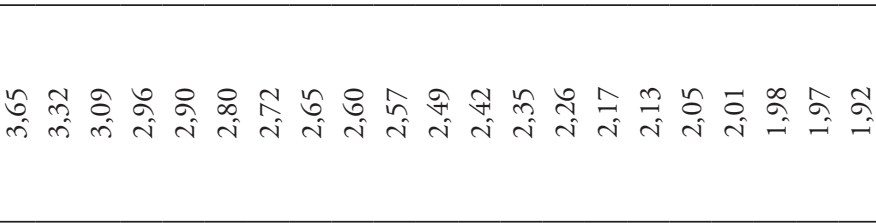 & \\
\hline 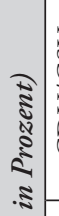 & & 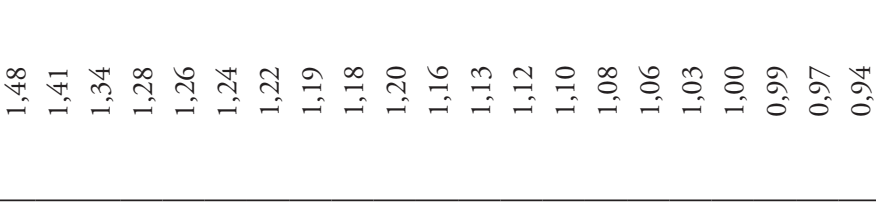 & \\
\hline 离. & & 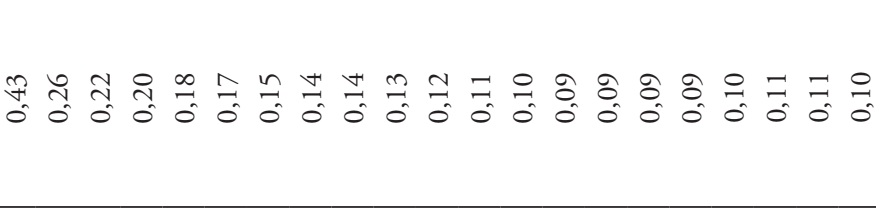 & \\
\hline 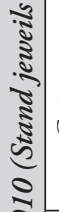 & & 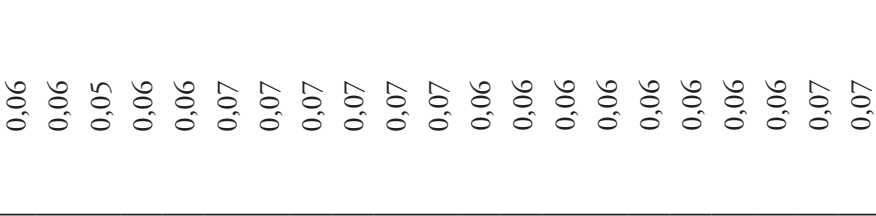 & \\
\hline 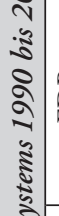 & 官: & 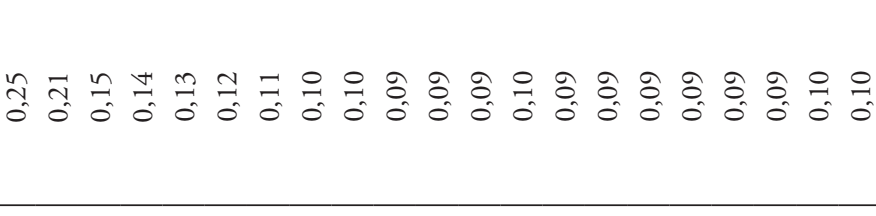 & \\
\hline 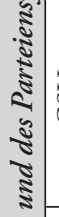 & כ. & 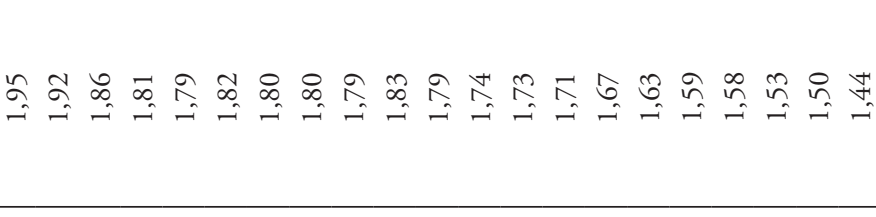 & \\
\hline 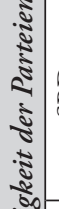 & $\hat{\omega}$ & 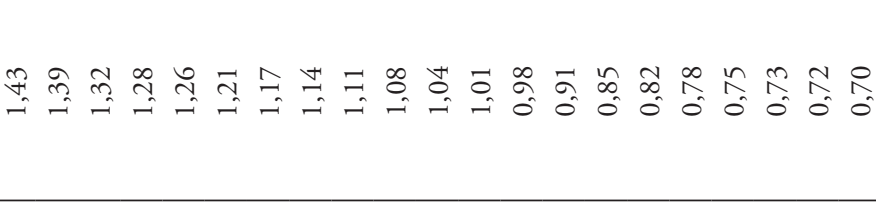 & \\
\hline 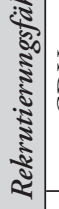 & & 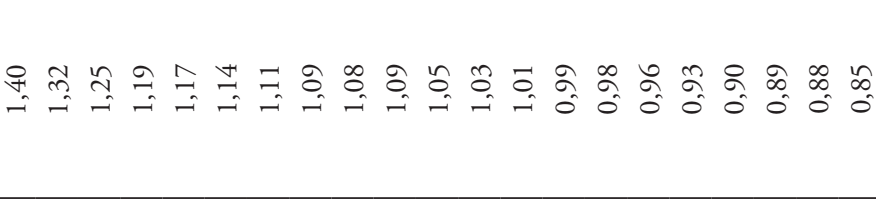 & \\
\hline $\begin{array}{l}\dot{v} \\
\stackrel{5}{\Xi} \\
\text { हैँ }\end{array}$ & & 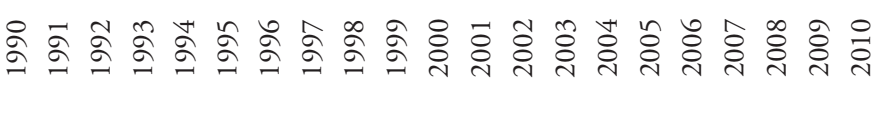 & \\
\hline
\end{tabular}




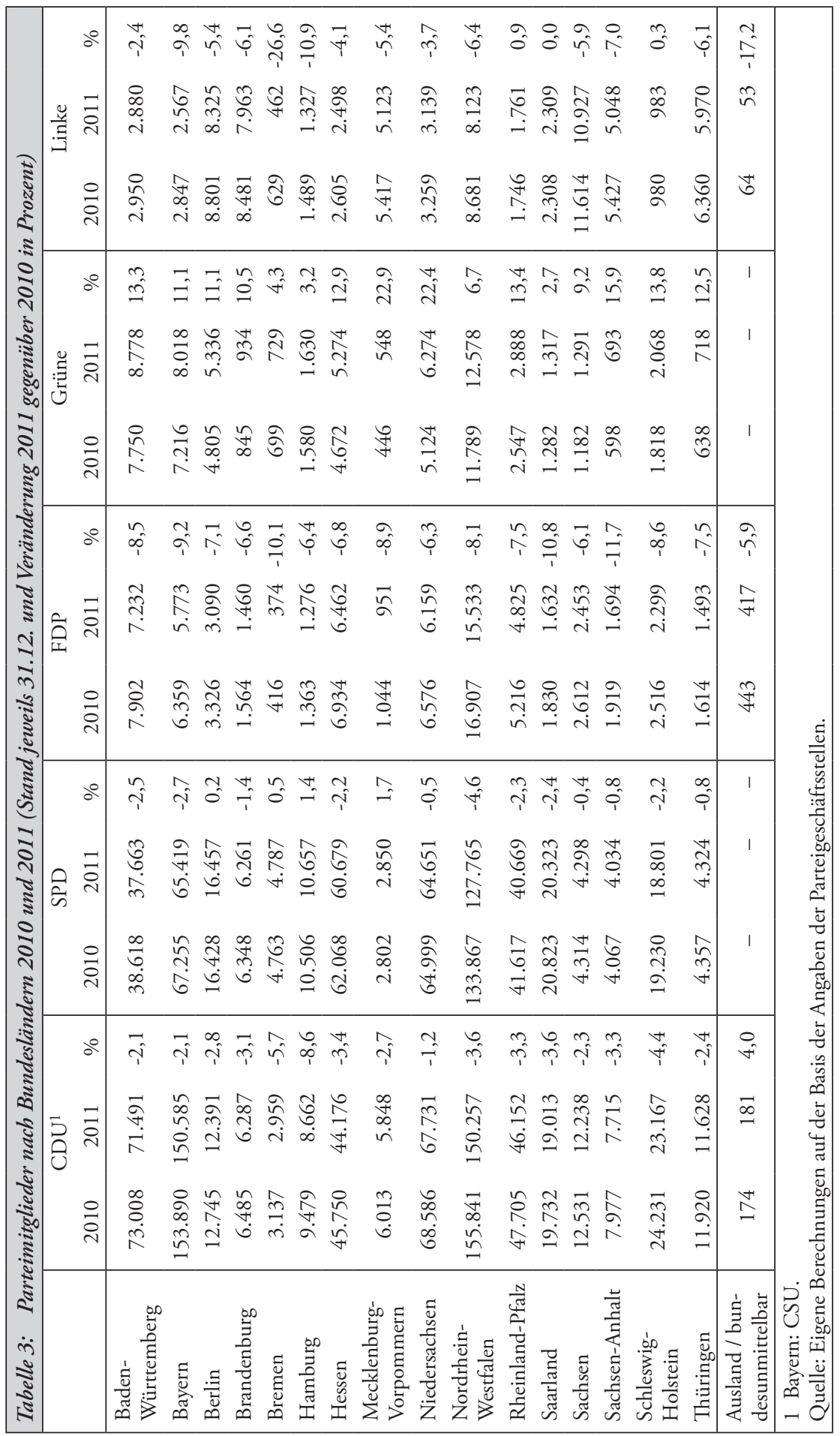




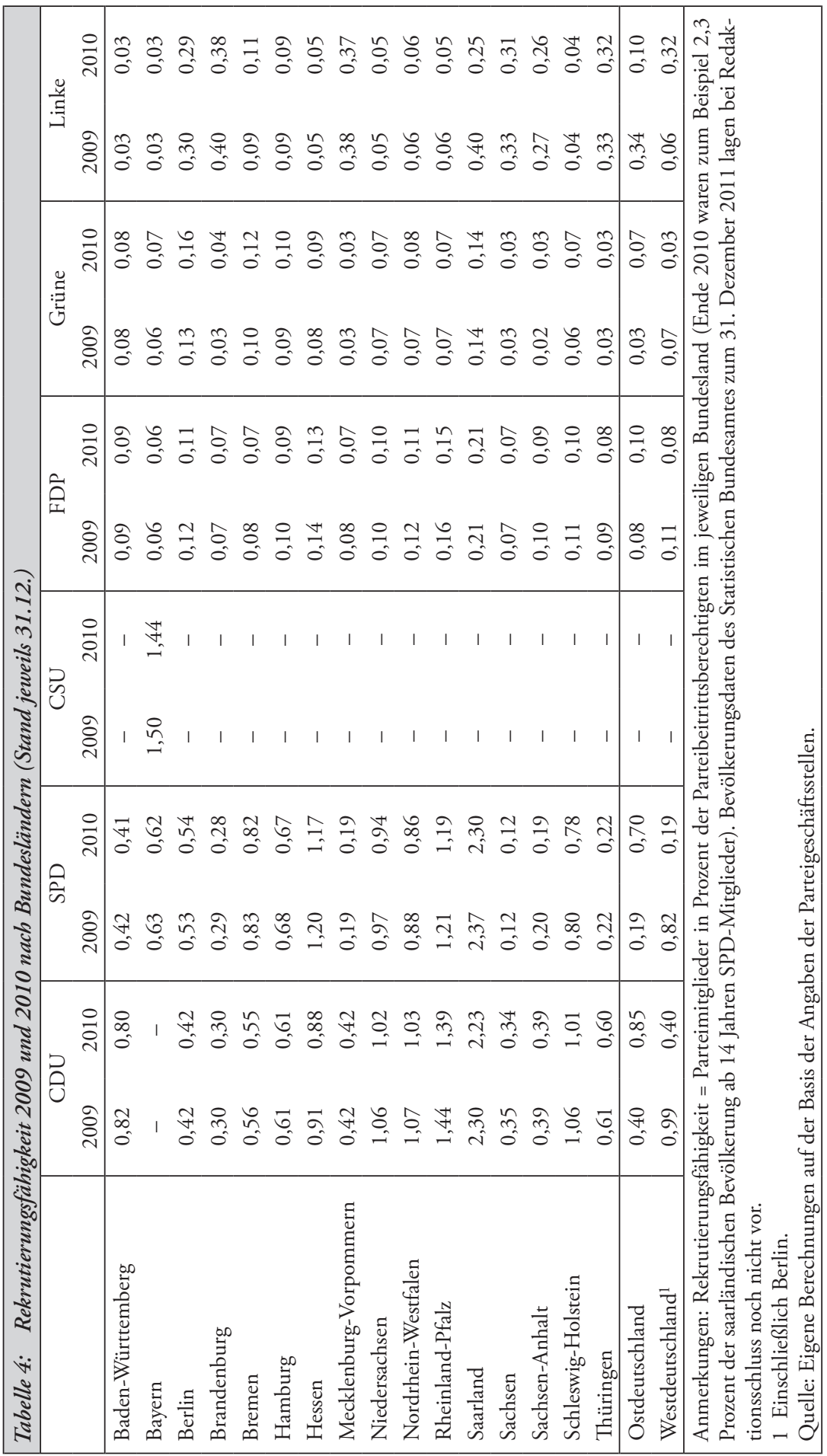




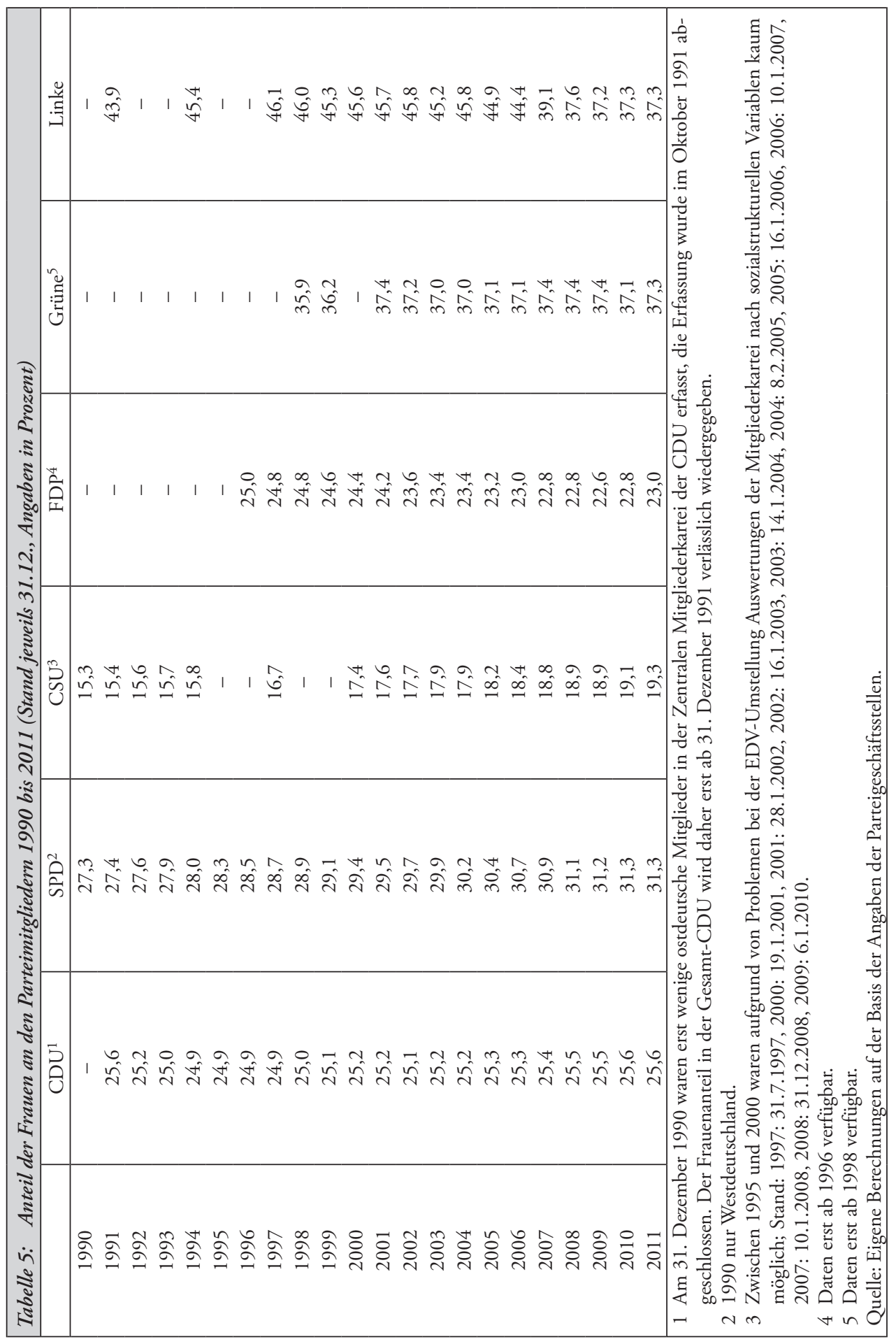




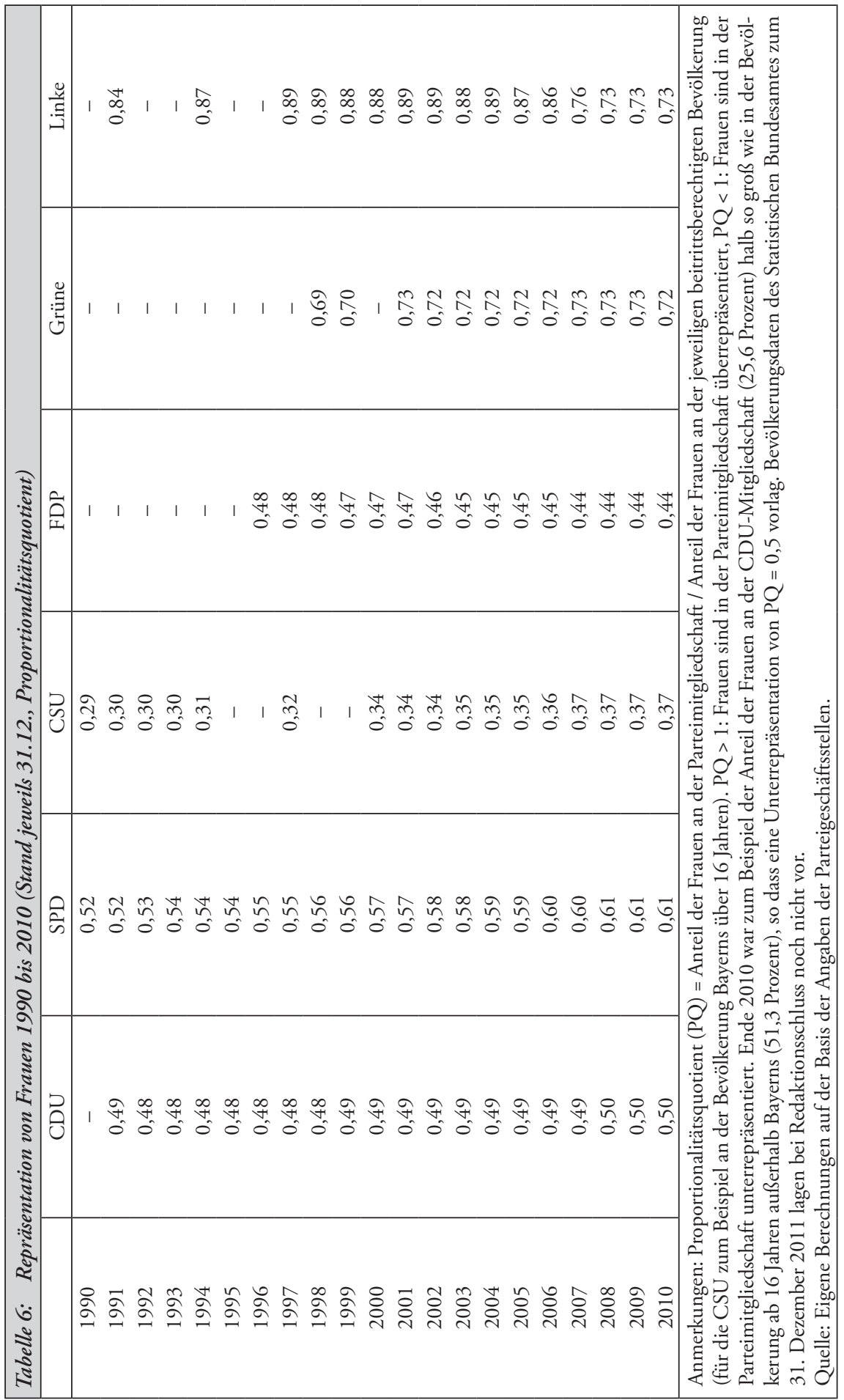




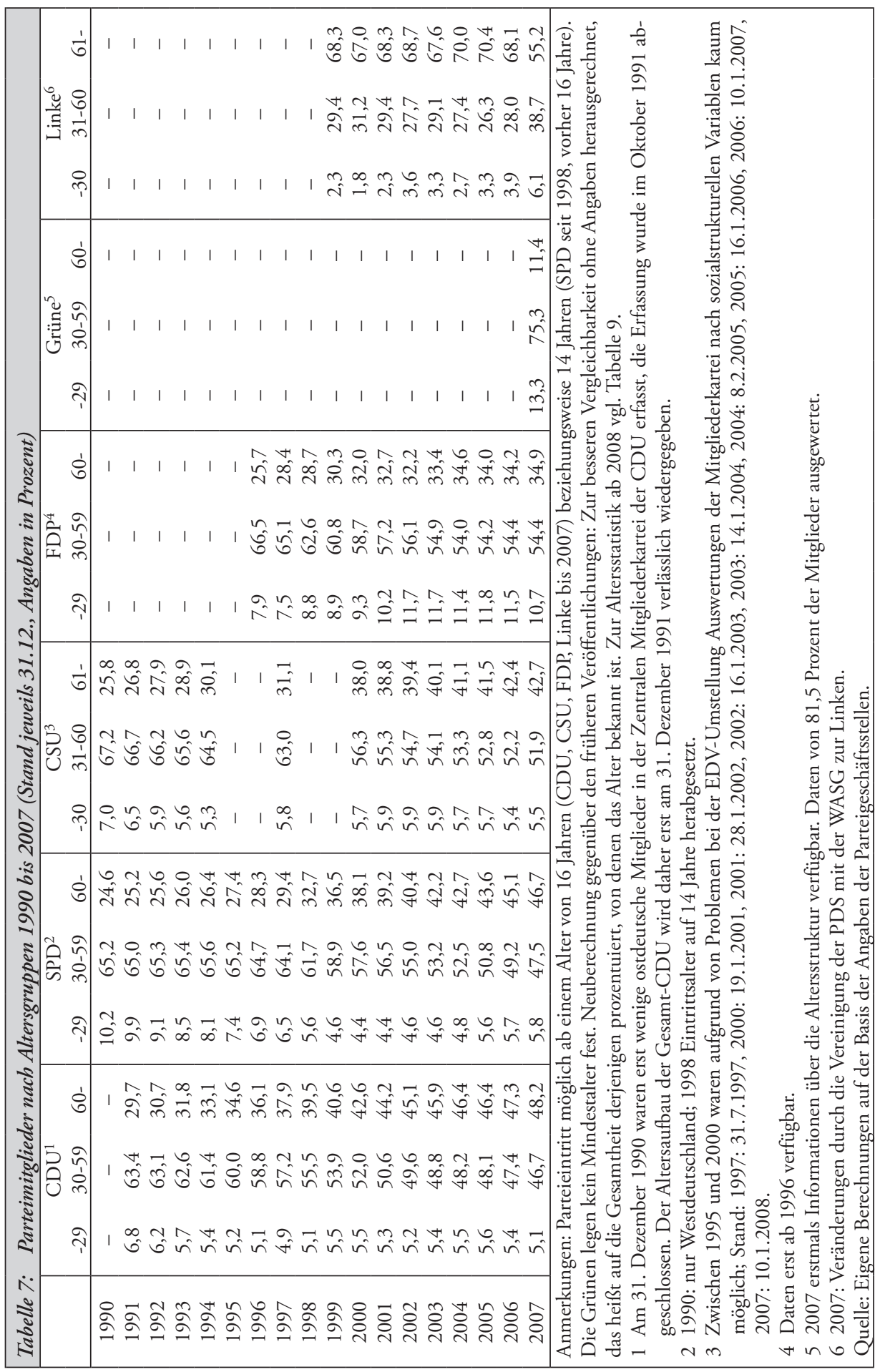




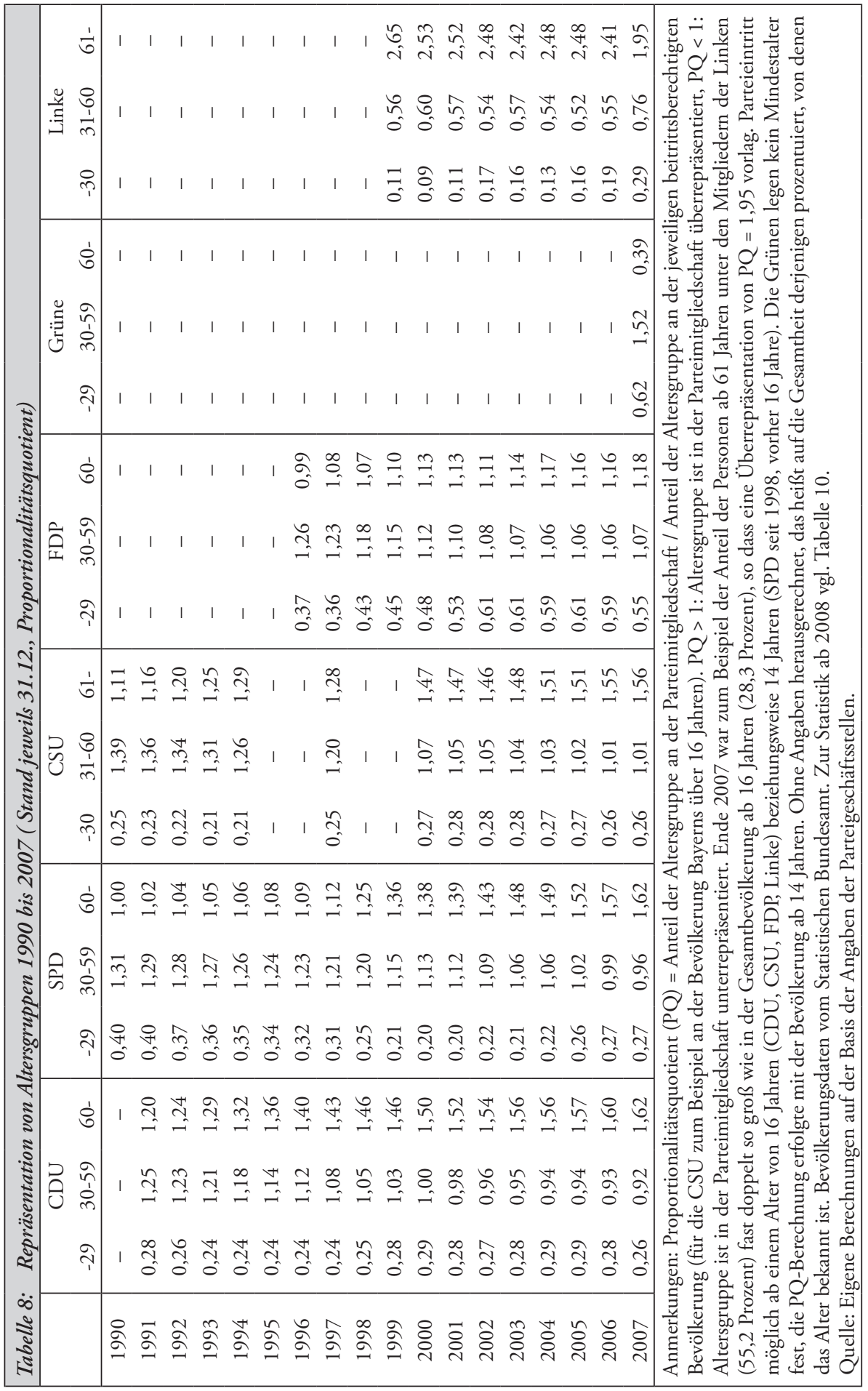




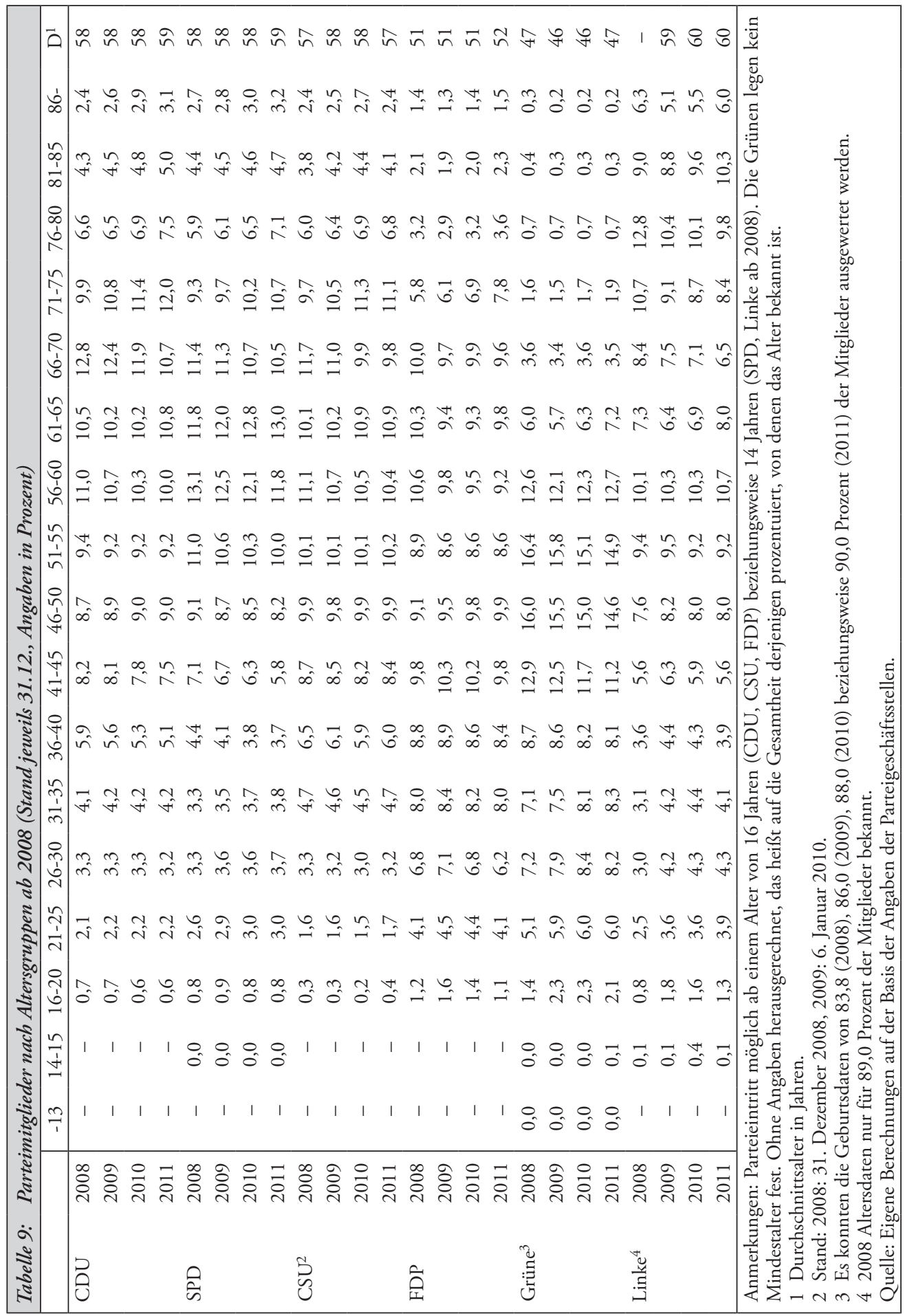




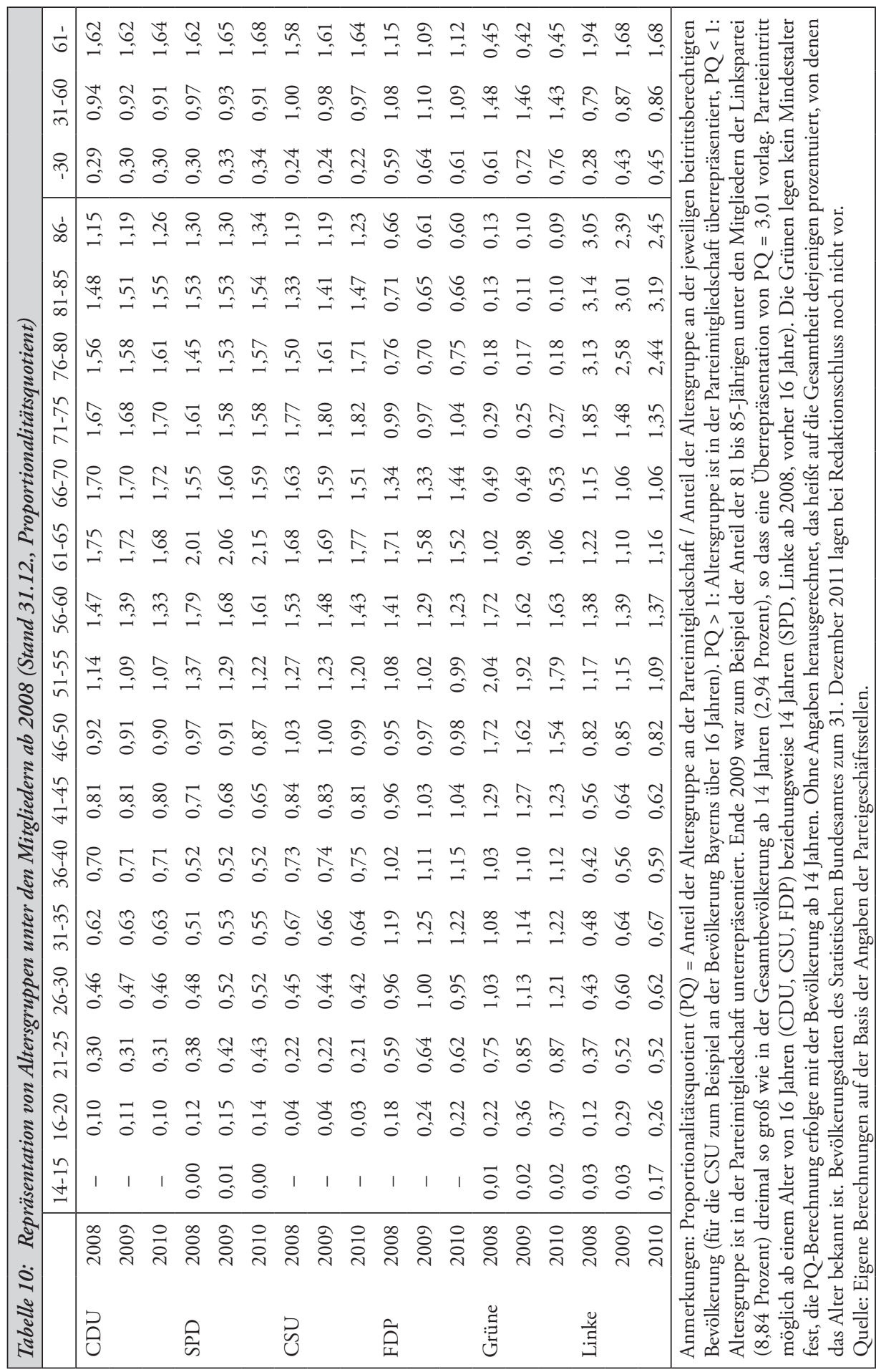




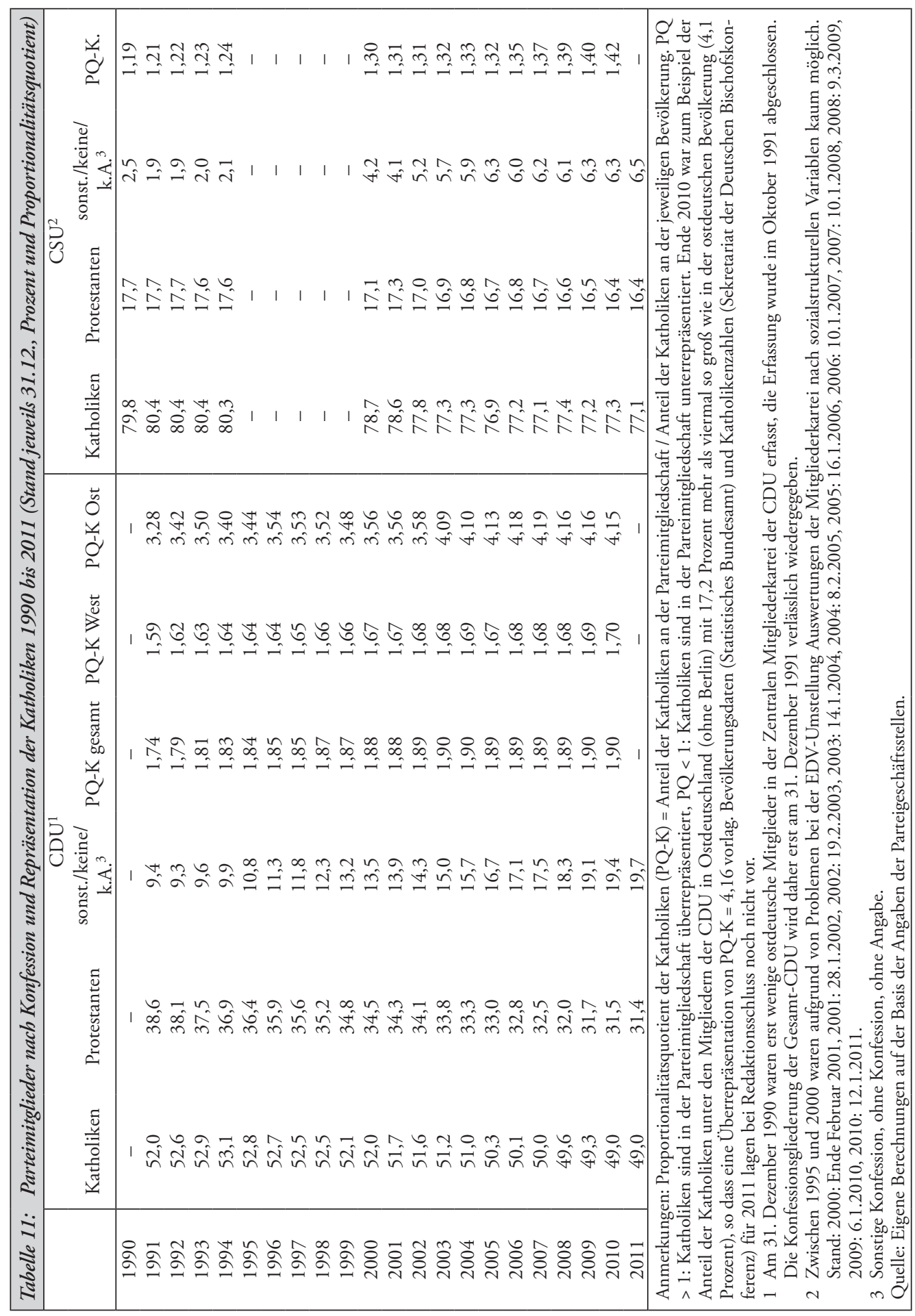




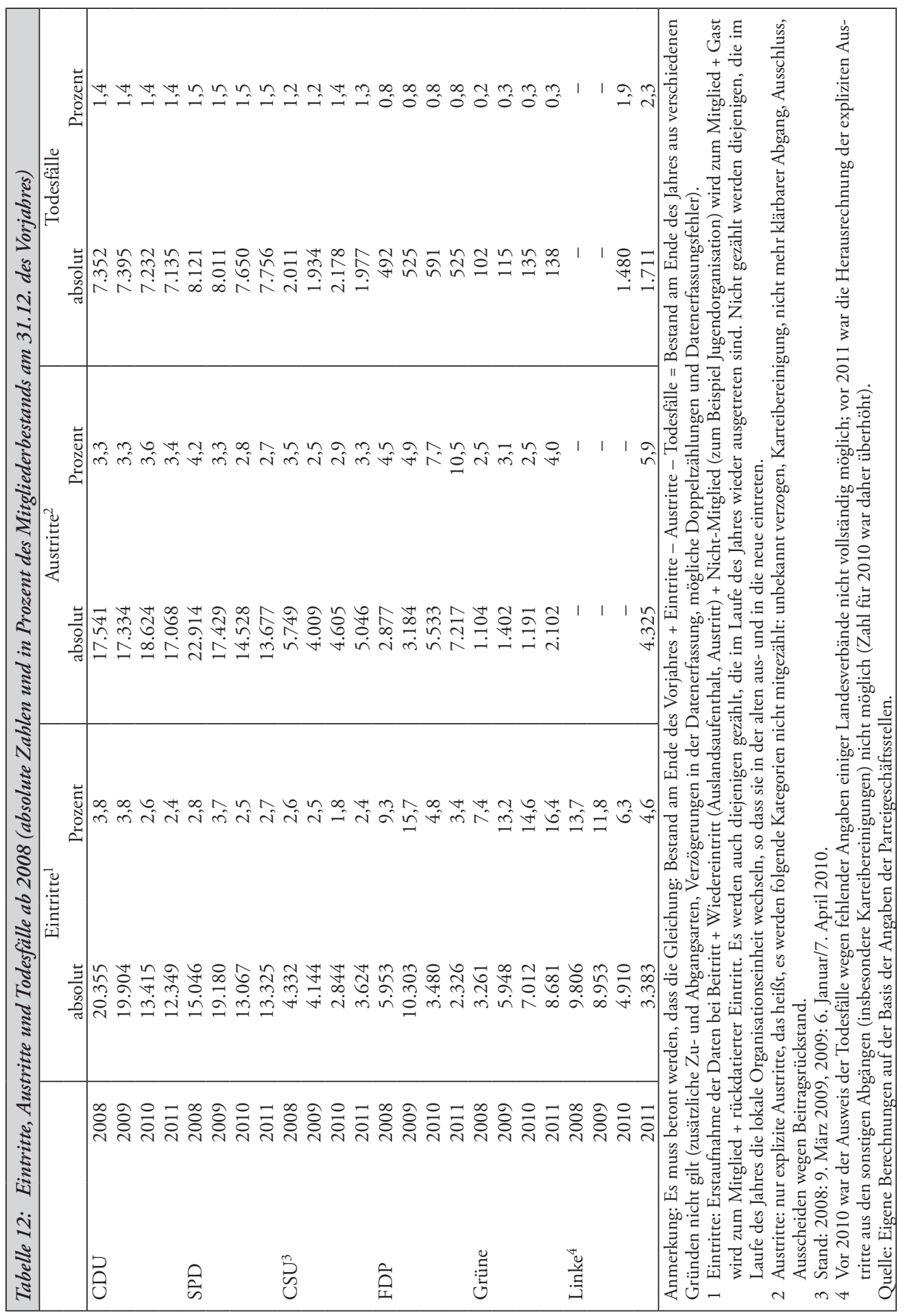




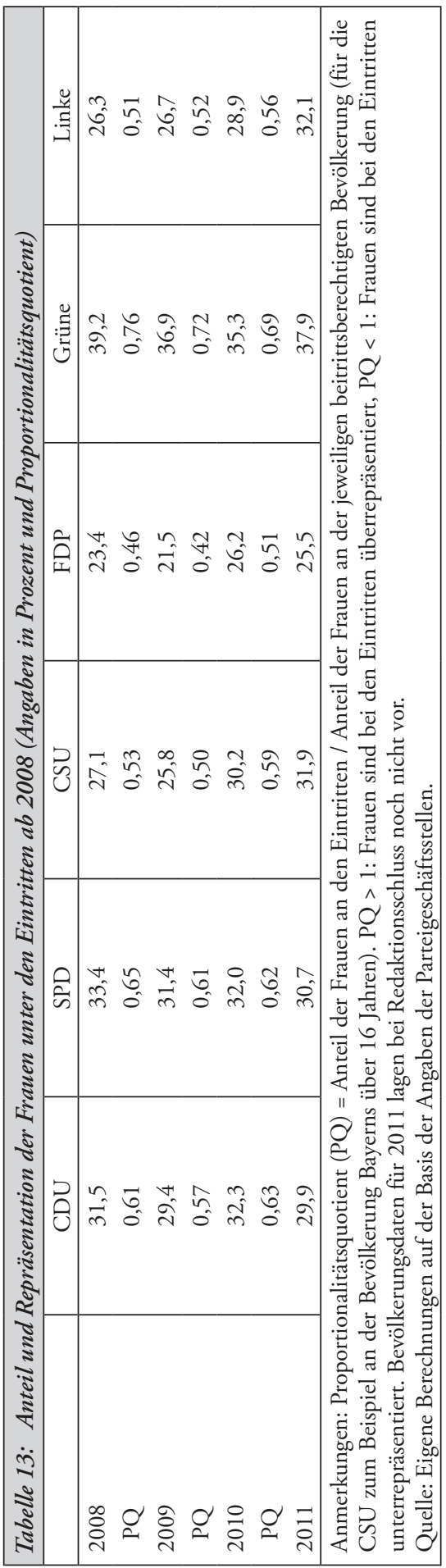




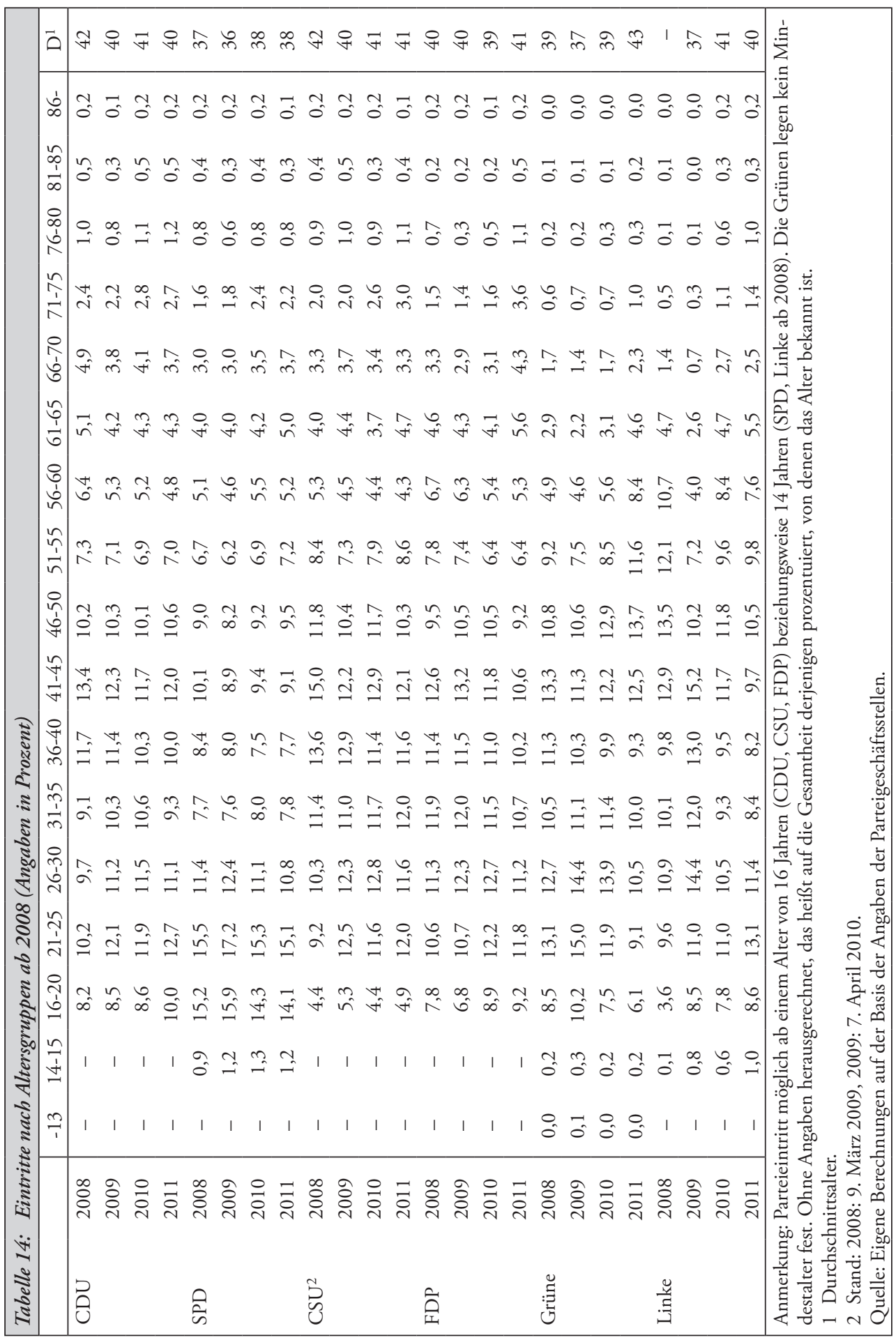




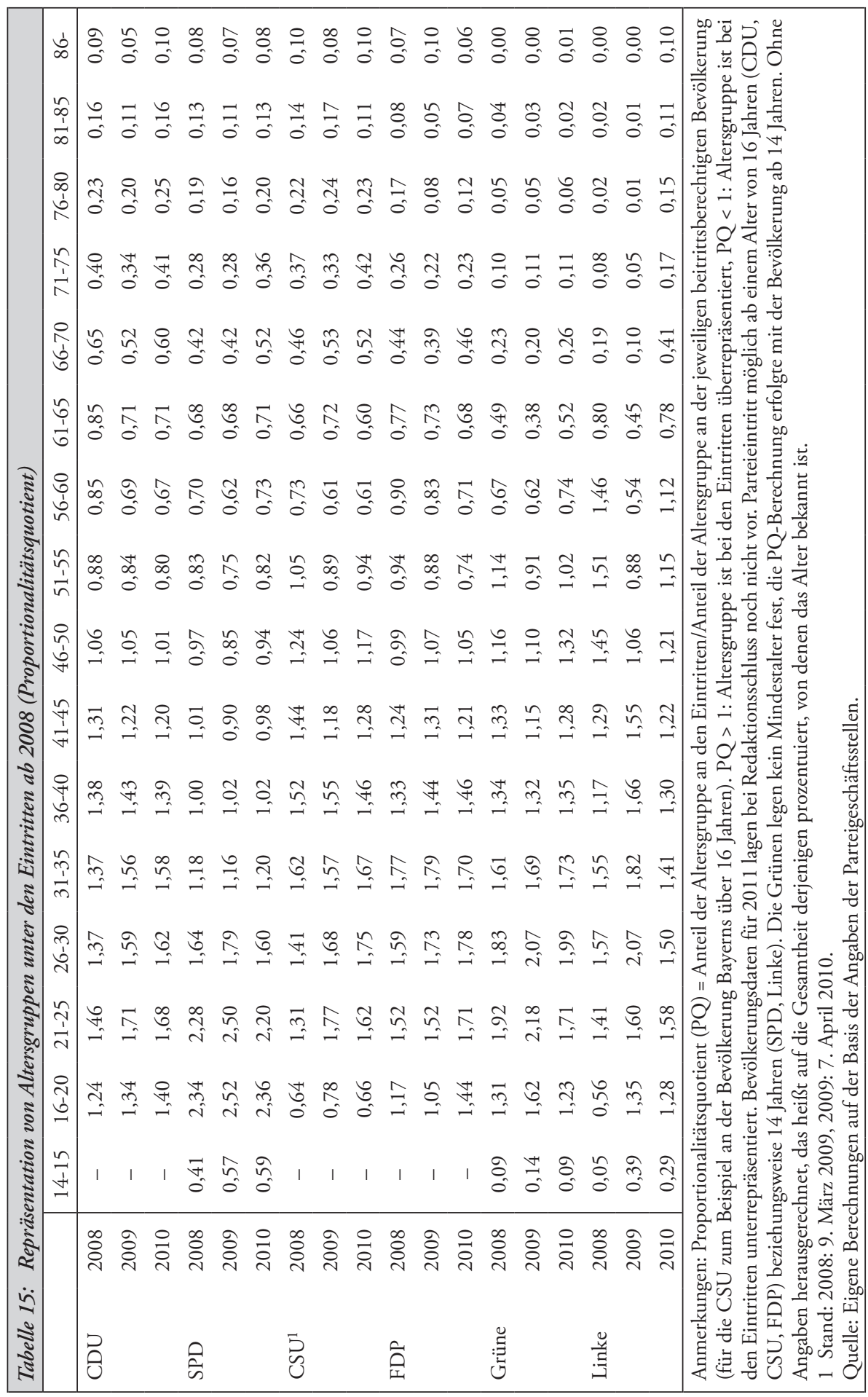

